\title{
Predictive value of the $M G M T$ promoter methylation status in metastatic melanoma patients receiving first-line temozolomide plus bevacizumab in the trial SAKK 50/07
}

\author{
PETER SCHRAML $^{1}$, ADRIANA VON TEICHMAN ${ }^{1}$, DANIELA MIHIC-PROBST ${ }^{1}$, \\ MATHEW SIMCOCK ${ }^{2}$, ADRIAN OCHSENBEIN ${ }^{3}$, REINHARD DUMMER $^{4}$, OLIVIER MICHIELIN $^{5}$, \\ BETTINA SEIFERT ${ }^{6}$, MARC SCHLÄPPI $^{7}$, HOLGER MOCH ${ }^{1 *}$ and ROGER VON MOOS ${ }^{8^{*}}$
}

\author{
${ }^{1}$ Institute of Surgical Pathology, University Hospital Zurich, Zurich; ${ }^{2}$ Swiss Group for Clinical Cancer Research (SAKK) \\ Coordinating Center; ${ }^{3}$ Inselspital, Bern; ${ }^{4}$ Clinic for Dermatology, University Hospital Zurich, Zurich; ${ }^{5} \mathrm{CHUV}$, Lausanne; \\ ${ }^{6}$ University of Basel, Basel; ${ }^{7}$ Canton's Hospital St. Gallen, St. Gallen; ${ }^{8}$ Canton's Hospital, Chur, Switzerland
}

Received April 7, 2012; Accepted April 30, 2012

DOI: $10.3892 /$ or.2012.1826

\begin{abstract}
The $\mathrm{O}^{6}$-methylguanine-DNA-methyltransferase $(M G M T)$ promoter methylation status is a predictive parameter for the response of malignant gliomas to alkylating agents such as temozolomide. First clinical trials with temozolomide plus bevacizumab therapy in metastatic melanoma patients are ongoing, although the predictive value of the $M G M T$ promoter methylation status in this setting remains unclear. We assessed MGMT promoter methylation in formalin-fixed, primary tumor tissue of metastatic melanoma patients treated with first-line temozolomide and bevacizumab from the trial $S A K K 50 / 07$ by methylation-specific polymerase chain reaction. In addition, the MGMT expression levels were also analyzed by MGMT immunohistochemistry. Eleven of 42 primary melanomas (26\%) revealed a methylated $M G M T$ promoter. Promoter methylation was significantly associated with response rates $\mathrm{CR}+\mathrm{PR}$ versus $\mathrm{SD}+\mathrm{PD}$ according to RECIST (response evaluation criteria in solid tumors) $(\mathrm{p}<0.05)$ with a trend to prolonged median progression-free survival (8.1 versus 3.4 months, $\mathrm{p}>0.05$ ). Immunohistochemically different protein expression patterns with heterogeneous and homogeneous nuclear MGMT expression were identified. Negative MGMT expression levels were associated with overall disease stabilization $\mathrm{CR}+\mathrm{PR}+\mathrm{SD}$ versus $\mathrm{PD}(\mathrm{p}=0.05)$. There was only a poor correlation between $M G M T$ methylation and lack of MGMT expression. A significant proportion of melanomas have a methylated $M G M T$ promoter. The $M G M T$ promoter
\end{abstract}

Correspondence to: Dr Roger von Moos, Medical Oncology, Canton's Hospital Graubünden, Loestrasse 170, CH-7000 Chur, Switzerland

E-mail: roger.vonmoos@ksgr.ch

*Shared senior authorship

Key words: melanoma, $\mathrm{O}^{6}$-methylguanine-DNA-methyltransferase, temozolomide, bevacizumab methylation status may be a promising predictive marker for temozolomide therapy in metastatic melanoma patients. Larger sample sizes may help to validate significant differences in survival type endpoints.

\section{Introduction}

The incidence of malignant melanoma is increasing worldwide. Stage IV melanoma is still a devastating disease with a median survival time of 6-9 months depending on the bulk and localisation of disease. Despite first successful immunotherapies as with ipililumab, a CTLA4 antibody in first and second line treatment of metastatic melanoma $(1,2)$ and targeted therapy for patients with BRAF mutations with PLX4032 $(3,4)$, there is still a need to better define patients responding to alkylating substances such as temozolomide especially in BRAF wild-type patients. Single agent dacarbazine has been the standard of care for many years. Response rates (RRs) of 5.5-13\% (5) have been reported in recent large phase III trials with a further $15-28 \%$ of patients having stable disease (SD), however, few responses are long-lasting (6).

Temozolomide is an oral alkylating agent with a mechanism of action similar to dacarbazine (7), that is spontaneously converted to its active metabolites (8). This oral drug has been used successfully in the treatment of primary brain tumors. It was shown that the gene promoter methylation status of the $\mathrm{O}^{6}$-methylguanine-DNA-methyltransferase gene $(M G M T)$ is of critical importance for survival in glioblastoma patients treated with temozolomide (9). MGMT silencing by promoter methylation impairs the ability of the MGMT protein to remove alkyl groups from the $\mathrm{O}^{6}$-position of guanine, thereby increasing the mutation rate in rapidly dividing tumor cells and improving patient outcome. Recently, we reported that about one-third of brain metastases from various origins including malignant melanoma revealed a methylated $M G M T$ promoter (10). Moreover, homogeneous MGMT immunoreactivity correlates with an unmethylated MGMT promoter status suggesting that brain metastases may be a potential target for therapy with alkylating substances. 
Temozolomide demonstrated clinical activity in a phase I trial with 4 out of 23 patients responding to treatment (11). This was supported by a phase II trial where $21 \%$ of the patients responded to treatment. A 9 months longer survival was seen in responders (12). Two large phase III randomized controlled trials of intravenous dacarbazine versus oral temozolomide every 4 weeks or dose dense every second week as first-line treatment in patients with melanoma demonstrated toxicity and response rate $(13,14)$ with a trend for superiority in progressionfree survival (PFS) and some quality of life domains in one trial in favour of temozolomide (13). A quite recently published phase III trial indicates that extended schedule escalated dose temozolomide has an acceptable safety profile, but does not improve overall survival (OS) and PFS in stage IV melanoma when compared to standard dose dacarbazine (14).

Blocking the vascular endothelial cell growth factor (VEGF) by bevacizumab showed significant survival advantage when combined with chemotherapy in advanced colorectal (15) and non-small cell lung cancer (16). The combination of bevacizumab and temozolomide was chosen in this trial because of the lack of overlapping toxicities. Furthermore, the combination of VEGF antibodies and chemotherapy improved survival in colon and lung cancer (15).

The optimal use of temozolomide in the treatment of metastatic melanoma is under discussion. The combined therapy of temozolomide and bevacizumab used in this phase II trial was an attempt to improve the response rate (17). A disease stabilization rate confirmed by independent external review of $52 \%$ at 12 weeks of the trial treatment is considered promising for further investigations. The response rate $(16.1 \%)$ as well as the PFS (4.2 months) obtained in our trial was slightly higher than reported for single agent temozolomide (9.8-12\%) and 2.1 months, respectively, despite having included also patients at higher risk (LDH >2 UNL; ECOG PS 2). Overall survival (9.3 months) in our trial was comparable to single agent temozolomide $(12,13,18)$.

The investigation of the methylation status of the $M G M T$ promoter was one of the prospectively defined translational research projects in this SAKK trial. Therefore, we tested $M G M T$ promoter methylation and MGMT protein expression in our patient set of SAKK 50/07 with metastatic melanoma to elucidate the molecular basis of tumor response and outcome in patients treated with temozolomide and bevacizumab.

\section{Material and methods}

Patients and tumors. Formalin-fixed, paraffin-embedded tissue samples of 62 patients previously untreated for metastatic melanoma with ECOG performance status $\leq 2$ were selected for $M G M T$ promoter methylation analysis and immunohistochemical analysis. The patients were treated with temozolomide at $150 \mathrm{mg} / \mathrm{m}^{2}$ days $1-7$ orally and bevacizumab at $10 \mathrm{mg} / \mathrm{kg}$ body weight day 1 intravenous every 2 weeks until disease progression or unacceptable toxicity. Response was assessed every 6 weeks by computed tomography according to the RECIST (response evaluation criteria in solid tumors) criteria.

The protocol was approved by local ethics review boards and all patients gave written informed consent. The trial was registered at the National Institute of Health (www.clinicaltrial. gov; identifier number: NCT00568048).
DNA extraction and methylation-specific PCR. All tumors were histologically reviewed by one pathologist (H.M.) on the basis of hematoxylin and eosin-stained tissue sections. One to three biopsy cylinders (0.6-mm diameter) were punched from tumor areas that contained at least $70 \%$ tumor cells. Genomic DNA extraction, sodium bisulfite modification of isolated DNA and the analysis of the methylation status of MGMT was done using a one-step PCR approach with 40 cycles and the nested primer pair described by Palmisano et al (19). DNA of normal lymphocytes and human colorectal adenocarcinoma cell-line SW620 was used as a negative and positive control for methylated alleles of $M G M T$, respectively.

Immunohistochemistry. Immunohistochemistry of melanoma sections was performed as described (10). Sections were incubated with clone MT3.1 (dilution 1:160; NeoMarkers, Newmarket, UK). MGMT-immunopositive cells revealed a strong nuclear staining. Lymphocytes and endothelial cells served as an internal positive control. The immunoreactivity was scored semi-quantitatively as follows: $0:<5 \%$ positive tumor cells, $1+$ : $5-75 \%$ positive tumor cells, $2+:>75-95 \%$ positive tumor cells, $3+:>95 \%$ positive tumor cells.

Statistical analyses. The endpoints of interest defined in the trial protocol and considered for this project include disease stabilization rate at week 12 (DSR12) consisting of either a complete response (CR), partial response (PR) or $\mathrm{SD}$, best overall response, PFS and OS. PFS was defined as the time from trial registration until either a disease progression or death, with patients censored at the time of starting a second line therapy or the last time they were known to be alive without progression. For survival-type endpoints subgroups were compared using the log-rank test. Associations between categorical variables were assessed by either the Chi-squared or Fisher's exact test as well as the Mantel-Haenszel Chi-square test of trend if a variable was ordinal. The data were analyzed in SAS (Statistical Analysis Systems, version 9.2).

\section{Results}

Patients. Between January 2008 and April 2009, 62 patients (40 male) were enrolled in the trial SAKK 50/07. None of the patients were ineligible or withdrew participation before treatment start. The median age at enrollment was 59 years (range: 29-82) and the median follow-up time was 20.1 (range: 1.7-32.6) months. All patients were administered at least one cycle of therapy. The independently reviewed DSR12 was 52\% including 10 patients with a PR and 22 patients with SD.

MGMT promoter methylation and protein expression. Tumor tissue was not available for 9 of the 62 patients enrolled in the trial. MGMT methylation status was reliably determined in 42 of $53(79 \%)$ formalin-fixed and paraffin-embedded samples. Overall, a methylated $M G M T$ promoter was detectable in 11 of $42(26 \%)$ of the metastatic melanomas by MS-PCR.

MGMT immunoreactivity was assessed in 49 of 53 (92\%) tumors. Of 53 tumors, 4 were excluded from further calculations because endothelial cells and/or leukocytes used as internal control for MGMT positivity were MGMT negative possibly indicating tissue fixation problems. Seventeen (35\%) tumor 


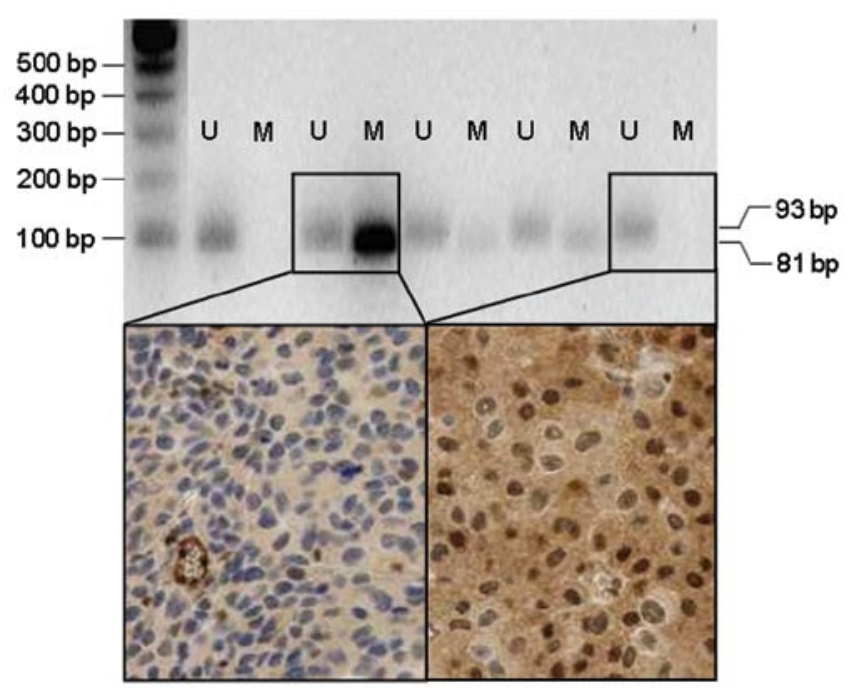

Figure 1. Methylation-specific PCR with 5 metastatic melanomas. U: unmethylated, M: methylated $M G M T$ (upper panel). Tumors with methylated $M G M T /$ no MGMT expression (left) and with unmethylated $M G M T /$ strong nuclear MGMT expression are highlighted.

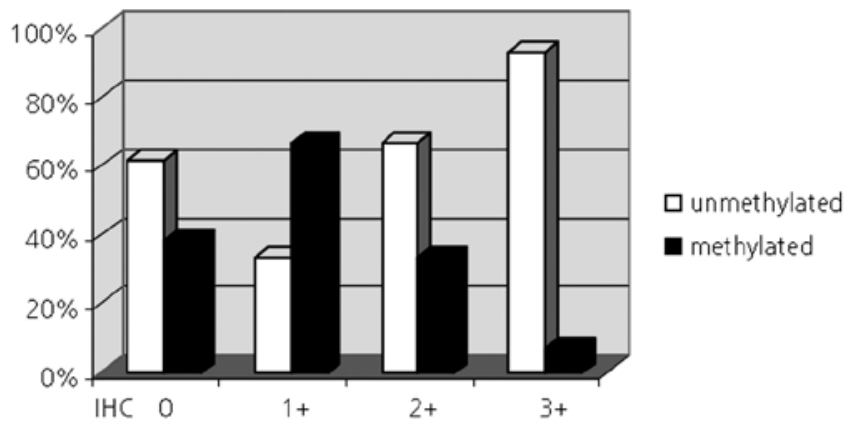

Figure 2. MGMT methylation status in the four MGMT expression subgroups. IHC 0: <5\%, IHC 1+: 5-75\%, IHC 2+: 75-95\%, IHC 3+: >95\% nuclear MGMT positive tumor cells.

samples were $3+, 11(22 \%)$ were $2+$, whereas $4(8 \%)$ were $1+$ and 17 (35\%) lacked immunoreactivity for MGMT.

Both the methylation status of the MGMT promoter as well as the MGMT immunoprofile were available in 39 patients. Examples of melanomas with unmethylated and methylated $M G M T$ promoter as well as with strongly positive and negative MGMT protein expression are shown in Fig. 1. No significant association was observed when the MGMT expression patterns in the methylated and in the unmethylated patient subgroups were compared. Of 28 (46\%) melanomas with an unmethylated MGMT promoter, 13 displayed a homogeneous 3+ MGMT immunoreactivity, 6 (21\%) tumors had a $2+$ score, one $(4 \%)$ had a $1+$ score and $8(29 \%)$ were MGMT negative. Within the subgroup of melanomas which had a methylated $M G M T$ promoter $5(45 \%)$ were also MGMT negative, two (18\%), three (27\%) and one (9\%) were scored $1+, 2+$ and $3+$, respectively (Fig. 2).

When we looked at the methylation status in the four MGMT expression subgroups we noted that in the patient subgroup with a $3+$ score 13 of $14(93 \%)$ tumors were also

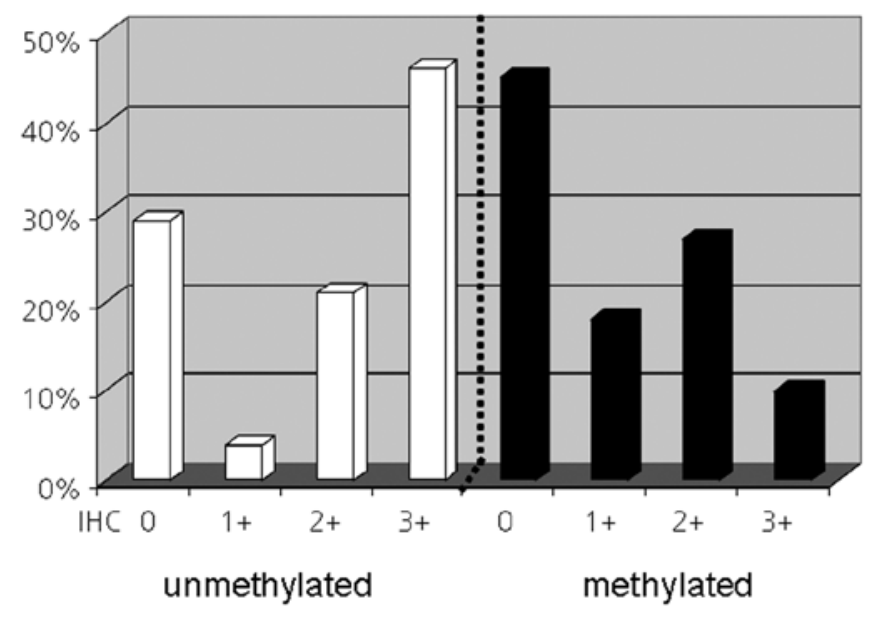

Figure 3. Association between $M G M T$ methylation and MGMT protein expression in metastatic melanoma. IHC 0: $<5 \%$, IHC 1+: 5-75\%, IHC 2+: 75-95\%, IHC 3+: >95\% nuclear MGMT positive tumor cells.

Table I. Association between response and MGMT promoter methylation status.

\begin{tabular}{lcc}
\hline $\begin{array}{l}\text { Methylation } \\
\text { status }\end{array}$ & Response & $\begin{array}{c}\text { Mantel-Haenszel } \\
\text { Chi-square test } \\
\text { of trend p-value }\end{array}$ \\
\hline Methylated vs. & CR, PR, SD, PD & 0.0803 \\
Unmethylated & CR + PR + SD, PD & 0.5573 \\
& CR + PR, SD + PD & 0.0426 \\
\hline
\end{tabular}

unmethylated. In contrast, no association was observed between MGMT negativity and promoter methylation. Only 5 of $13(38 \%)$ MGMT negative tumors had a methylated promoter (Fig. 3). However, no statistical correlation existed between expression and methylation status.

MGMT promoter methylation status and patient outcome. Patients with methylated $M G M T$ promoter had higher response rates according to RECIST, when CR + PR versus SD + PD were compared $(p=0.0426$, Table I). No significant differences were obtained for other response groupings.

PFS and OS were stratified by MGMT methylation status. Median PFS time in the methylated subgroup was 8.1 months [95\% confidence interval $(\mathrm{CI}): 1.7,8.4]$ versus 3.4 months (95\% CI: 1.4, 5.4) in the unmethylated subgroup. Median OS time in the methylated subgroup was 9.9 months $(95 \%$ CI: 8.4, $15.9)$ versus 9.1 months $(95 \%$ CI: $6.6,11.9)$ in the unmethylated subgroup. Although, in the methylated subgroup median PFS was exceeded by more than 4 months compared to the unmethylated subgroup the results did not reach statistical significance.

MGMT protein expression and patient outcome. The different groupings for the MGMT protein expression (0-5, 5-75, 75-95 and $95-100 \%$ nuclear positive tumor cells) were compared in various groupings with regard to best overall response. The best response outcome was obtained from patients that were 
Table II. Best overall response by MGMT protein expression and promoter methylation status.

\begin{tabular}{|c|c|c|c|c|c|c|}
\hline \multirow[b]{2}{*}{ Methylation status } & \multirow[b]{2}{*}{ Best response } & \multicolumn{5}{|c|}{ MGMT expression (\% positive tumor cells) } \\
\hline & & Unknown & $0-5 \%$ & $5-75 \%$ & $75-95 \%$ & $95-100 \%$ \\
\hline \multirow[t]{5}{*}{ Methylated } & Complete response & - & - & - & - & - \\
\hline & Partial response & - & 5 & - & - & 1 \\
\hline & Stable disease & - & - & 1 & 2 & - \\
\hline & Disease progression & - & - & 1 & 1 & - \\
\hline & Unkown & - & - & - & - & - \\
\hline \multirow[t]{5}{*}{ Unmethylated } & Complete response & - & - & - & - & 1 \\
\hline & Partial response & - & 1 & - & 2 & 1 \\
\hline & Stable disease & 2 & 4 & - & 3 & 6 \\
\hline & Disease progression & 1 & 2 & 1 & 1 & 5 \\
\hline & Unknown & - & 1 & - & - & - \\
\hline \multirow[t]{5}{*}{ Unknown } & Complete response & & & & & \\
\hline & Partial response & 2 & 1 & - & - & 1 \\
\hline & Stable disease & 3 & 3 & - & - & 2 \\
\hline & Disease progression & 1 & 1 & 2 & 2 & 2 \\
\hline & Unknown & - & - & - & - & - \\
\hline
\end{tabular}

MGMT negative $(<5 \%)$ versus otherwise when $\mathrm{CR}+\mathrm{PR}+\mathrm{SD}$ versus PD were compared $(\mathrm{p}=0.05)$.

PFS and OS were stratified by the MGMT expression groups. In general, patients with negative or heterogeneous MGMT expression had longer PFS and OS rates than patients with high and homogeneous MGMT expression. However, these differences did not reach statistical significance (data not shown). Best overall response by MGMT protein level and promoter methylation status is summarized in Table II.

\section{Discussion}

We investigated the epigenetic silencing of $M G M T$ by both $M G M T$ promoter methylation and the MGMT protein expression status in metastatic melanomas to assess the predictive value for benefit from temozolomide in combination with bevacizumab. In our study, $26 \%$ of metastatic melanomas revealed a methylated $M G M T$ promoter. This result is in line with previous studies on similar patient cohorts resulting in MGMT promoter methylation between 20 and 31\% $(10,20,21)$. Of note, a methylated $M G M T$ promoter correlated significantly with complete and partial responses. Compared to patients with unmethylated $M G M T$ promoter this patient group had also a trend to prolonged median PFS and OS of more than 4 months.

Our combined analysis of $M G M T$ promoter methylation status and protein expression is unique and revealed a strong association between homogeneous MGMT expression and unmethylated MGMT promoter. Ninety-three percent of the tumors with $>95 \%$ MGMT-positive tumor cells had an unmethylated MGMT promoter. In contrast, only $44 \%$ of the tumors with no or heterogeneous MGMT positivity had a methylated MGMT promoter. Similar results were observed in patients with brain metastases of various solid tumors, as previously published (10).

There are several possible explanations for this discrepancy: i) gene mutations may lead to loss of functional MGMT expression independent of the $M G M T$ promoter methylation status; ii) in a certain set of unmethylated and MGMT negative melanomas, $M G M T$ may be induced after addition of alkylating, DNA damaging agents $(22,23)$; iii) both discordance between $M G M T$ promoter methylation and individual metastases from the same patient (20) and/or intra-tumoral heterogeneity of promoter hypermethylation (21) may significantly weaken $M G M T$ methylation as a reliable predictor of chemosensitivity; iv) some tumors may be interpreted unmethylated because the corresponding PCR product is not detectable or not reproducible. In contrast to MS-PCR, pyrosequencing enables to identify promoter regions methylated from 0 to $100 \%$. A previous melanoma study showed that in $50 \%$ of the tumors, MGMT promoter methylation was $10 \%$ or less (18). It is therefore tempting to speculate that tumors with low MGMT methylation are considered unmethylated when MS-PCR is used.

In a recently published study methylation of the $M G M T$ promoter was analyzed in metastatic melanoma with the combined bisulfite restriction analysis technique (24). In contrast to our findings, those patients with a methylated $M G M T$ promoter neither had a survival advantage nor a better response rate but suffered from more severe adverse events compared to those with an unmethylated promoter. One possible explanation for the discrepant outcomes may be the difference in treatment. The lack of MGMT in temozolomide-treated melanoma cells causes an increase of toxicity, which may even be enhanced in the presence of bevacizumab by attenuating vascularization and therefore oxygen and energy supply in the tumor. 
We also believe that the use of different methods and the analysis of different $\mathrm{CpG}$ regions within the MGMT promoter may lead to contradictory results. Consequently, a standardized protocol with a still to be defined threshold that more reliably separates $M G M T$ methylated (inactive) from unmethylated (active) melanoma patients would be highly desirable. Here we selected to use a protocol which was previously described for investigating the $M G M T$ methylation status in glioma patients (9) and which allowed us to directly compare the results obtained from melanoma and glioma patients.

A reproducible and reliable promoter methylation analysis was only possible in 42 of 53 cases. DNA treated with formalin and bisulfite is highly modified and degraded and thus of limited quality to perform reliable PCR. To minimize the amplification of artificial PCR products we used a one-step PCR approach. The success rate for DNA amplification was much higher compared to that obtained from a recent study in which nested PCR with 80 cycles was used (10). All 53 melanoma DNA samples were amplifiable, the results of $11(19 \%)$ cases, however, were not reproducible. Similar error rates were reported in the literature $(10,25,26)$. The reasons why some of the DNA samples extracted from formalin fixed tissue are not suitable for MS-PCR may be a too long tissue fixation time, the use of unbuffered formalin or insufficient dehydration of the tissue.

Based on our data we suggest that both molecular and immunohistochemical approaches may be helpful to identify responders (methylated) as well as non-responders (unmethylated and homogeneous expression). For routine diagnosis further optimized standard protocols with preferably unfixed, snap-frozen biopsies are necessary to improve specificity and sensitivity.

\section{Acknowledgements}

We thank Sonja Schmid, Martina Storz and Silvia Behnke for their excellent technical assistance. This work was supported by Roche Pharma (Schweiz) AG, Schering Plough, Essex, UK and the Swiss State Secretariat for Education and Research.

\section{References}

1. Hodi FS, O'Day SJ, McDermott DF, et al: Improved survival with ipilimumab in patients with metastatic melanoma. N Engl J Med 363: 711-723, 2010.

2. Robert C, Thomas L, Bondarenko I, et al: Ipilimumab plus dacarbazine for previously untreated metastatic melanoma. $\mathrm{N}$ Engl J Med 364: 2517-2526, 2011.

3. Chapman PB, Hauschild A, Robert C, et al: Improved survival with vemurafenib in melanoma with BRAF V600E mutation. N Engl J Med 364: 2507-2516, 2011.

4. Flaherty KT, Puzanov I, Kim KB, et al: Inhibition of mutated, activated BRAF in metastatic melanoma. N Engl J Med 363 : 809-819, 2010

5. Bedikian AY, Millward M, Pehamberger $\mathrm{H}$, et al: Bcl-2 antisense (oblimersen sodium) plus dacarbazine in patients with advanced melanoma: the Oblimersen Melanoma Study Group. J Clin Oncol 24: 4738-4745, 2006.

6. Chapman PB, Einhorn LH, Meyers ML, et al: Phase III multicenter randomized trial of the Dartmouth regimen versus dacarbazine in patients with metastatic melanoma. J Clin Oncol 17: 2745-2751, 1999.

7. Stevens MF, Hickman JA, Langdon SP, et al: Antitumor activity and pharmacokinetics in mice of 8-carbamoyl-3-methylimidazo[5,1-d]-1,2,3,5-tetrazin-4(3H)-one (CCRG 81045; M\&B 39831 ), a novel drug with potential as an alternative to dacarbazine. Cancer Res 47: 5846-5852, 1987.
8. Tsang LL, Quarterman CP, Gescher A and Slack JA: Comparison of the cytotoxicity in vitro of temozolomide and dacarbazine, prodrugs of 3-methyl-(triazen-1-yl)imidazole-4-carboxamide. Cancer Chemother Pharmacol 27: 342-346, 1991.

9. Hegi ME, Diserens AC, Gorlia T, et al: MGMT gene silencing and benefit from temozolomide in glioblastoma. $\mathrm{N}$ Engl J Med 352: 997-1003, 2005.

10. Ingold B, Schraml P, Heppner FL and Moch H: Homogeneous MGMT immunoreactivity correlates with an unmethylated MGMT promoter status in brain metastases of various solid tumors. PLoS One 4: e4775, 2009.

11. Newlands ES, Blackledge GR, Slack JA, et al: Phase I trial of temozolomide (CCRG 81045: M\&B 39831: NSC 362856). Br J Cancer 65: 287-291, 1992.

12. Bleehen NM, Newlands ES, Lee SM, et al: Cancer Research Campaign phase II trial of temozolomide in metastatic melanoma. J Clin Oncol 13: 910-913, 1995.

13. Middleton MR, Grob JJ, Aaronson N, et al: Randomized phase III study of temozolomide versus dacarbazine in the treatment of patients with advanced metastatic malignant melanoma. J Clin Oncol 18: 158-166, 2000.

14. Patel PM, Suciu S, Mortier L, et al: Extended schedule, escalated dose temozolomide versus dacarbazine in stage IV melanoma: Final results of a randomised phase III study (EORTC 18032). Eur J Cancer 47: 1476-1483, 2011.

15. Kabbinavar F, Hurwitz HI, Fehrenbacher L, et al: Phase II, randomized trial comparing bevacizumab plus fluorouracil (FU)/leucovorin (LV) with FU/LV alone in patients with metastatic colorectal cancer. J Clin Oncol 21: 60-65, 2003.

16. Johnson DH, Fehrenbacher L, Novotny WF, et al: Randomized phase II trial comparing bevacizumab plus carboplatin and paclitaxel with carboplatin and paclitaxel alone in previously untreated locally advanced or metastatic non-small-cell lung cancer. J Clin Oncol 22: 2184-2191, 2004.

17. von Moos R, Seifert B, Simcock M, et al: First-line temozolomide combined with bevacizumab in metastatic melanoma: a multicentre phase II trial (SAKK 50/07). Ann Oncol 23: 531-536, 2011.

18. Rietschel P, Wolchok JD, Krown S, et al: Phase II study of extended-dose temozolomide in patients with melanoma. J Clin Oncol 26: 2299-2304, 2008.

19. Palmisano WA, Divine KK, Saccomanno G, et al: Predicting lung cancer by detecting aberrant promoter methylation in sputum. Cancer Res 60: 5954-5958, 2000.

20. Kohonen-Corish MR, Cooper WA, Saab J, Thompson JF, Tren RJ and Millward MJ: Promoter hypermethylation of the $\mathrm{O}(6)$-methylguanine DNA methyltransferase gene and microsatellite instability in metastatic melanoma. J Invest Dermatol 126: 167-171, 2006.

21. Rastetter M, Schagdarsurengin U, Lahtz C, et al: Frequent intra-tumoural heterogeneity of promoter hypermethylation in malignant melanoma. Histol Histopathol 22: 1005-1015, 2007.

22. Fritz G, Tano K, Mitra S and Kaina B: Inducibility of the DNA repair gene encoding $\mathrm{O}^{6}$-methylguanine-DNA methyltransferase in mammalian cells by DNA-damaging treatments. Mol Cell Biol 11: 4660-4668, 1991.

23. Grombacher T, Mitra S and Kaina B: Induction of the alkyltransferase (MGMT) gene by DNA damaging agents and the glucocorticoid dexamethasone and comparison with the response of base excision repair genes. Carcinogenesis 17: 2329-2336, 1996.

24. Hassel JC, Sucker A, Edler L, et al: MGMT gene promoter methylation correlates with tolerance of temozolomide treatment in melanoma but not with clinical outcome. Br J Cancer 103: 820-826, 2010.

25. Hamilton MG, Roldan G, Magliocco A, McIntyre JB, Parney I and Easaw JC: Determination of the methylation status of MGMT in different regions within glioblastoma multiforme. J Neurooncol 102: 255-260, 2011.

26. Preusser M, Elezi L and Hainfellner JA: Reliability and reproducibility of PCR-based testing of $\mathrm{O}^{6}$-methylguanine-DNA methyltransferase gene (MGMT) promoter methylation status in formalin-fixed and paraffin-embedded neurosurgical biopsy specimens. Clin Neuropathol 27: 388-390, 2008. 